\title{
Le rapport à littérature et à son enseignement : Comprendre ce qui s'enseigne sous le nom de littérature
}

\section{Ana Dias-Chiaruttini}

\section{QpenEdition}

\section{Journals}

Édition électronique

URL : http://journals.openedition.org/trema/4620

DOI : $10.4000 /$ trema.4620

ISSN : 2107-0997

Éditeur

Faculté d'Éducation de l'université de Montpellier

Édition imprimée

Date de publication : 1 octobre 2018

Pagination : $5-16$

ISBN : 979-10-96627-05-9

ISSN : 1167-315X

\section{Référence électronique}

Ana Dias-Chiaruttini, « Le rapport à littérature et à son enseignement : Comprendre ce qui s'enseigne sous le nom de littérature », Tréma [En ligne], 49 | 2018, mis en ligne le 15 octobre 2018, consulté le 22 avril 2019. URL : http://journals.openedition.org/trema/4620 ; DOI : 10.4000/trema.4620

Ce document a été généré automatiquement le 22 avril 2019

Trema 


\title{
Le rapport à littérature et à son enseignement : Comprendre ce qui s'enseigne sous le nom de littérature
}

\author{
Ana Dias-Chiaruttini
}

En situation didactique, ce que nous allons apercevoir, ce n'est pas ce que le professeur sait, mais ce qu'il fait de ce qu'il sait, ce jour-là, à cette heure-là, avec ce groupe-là.

Blanchard-Laville (2013, p. 136)

\section{Introduction}

1 Je propose dans le cadre de cet article de revenir sur le concept rapport à et à la façon dont je le mobilise dans mes travaux, depuis plus de 10 ans. Si le concept est essentiellement issu de travaux en sociologie, il a également été formalisé par les didactiques, comme le montre notamment la notice dont il fait l'objet dans le Dictionnaire des concepts fondamentaux en didactiques dirigé par Y. Reuter (2007/2010/2013). En didactique du français, le recours à ce concept a permis des travaux importants notamment, en France, en didactique de l'écriture (Barré de Miniac, 2000). Au Québec, il est convoqué par des chercheurs qui s'intéressent à la culture des enseignants (Falardeau \& Simard, 2007/2011) ou à la construction de leur professionnalité notamment dans l'enseignement de la lecture littéraire (Émery-Bruneau, 2010).

2 Cet écrit de travail vise à clarifier un usage du concept au sein de mes travaux. Ces derniers se situent en didactique du français et portent sur l'enseignement de la littérature, et la façon dont celui-ci participe à la configuration de la discipline français, selon les degrés et les contextes sociologiques d'enseignement. Ma réflexion englobe une perspective diachronique pour comprendre en partie comment cet enseignement évolue. La littérature est pour moi une composante de la discipline français. Elle contribue à formaliser et à organiser une partie des contenus associés à la discipline scolaire. Je 
cherche à reconstruire ce qui s'enseigne sous le nom de littérature, à travers la réception des textes rendue possible par des situations spécifiques (telle que le débat interprétatif, Dias-Chiaruttini, 2015). C'est l'actualisation de cet enseignement au sein de la discipline français qu'il m'intéresse d'analyser. Les pratiques enseignantes prennent, dans ce projet scientifique, une place importante que je reconstruis à travers des déclarations de pratiques et des observations de pratiques effectives. C'est dans cette perspective que je convoque le concept rapport à... afin d'étudier les rapports à la littérature et à son enseignement (RL\&E ). Ceux-ci me permettent à la fois de mieux comprendre les pratiques déclarées et observées et d'identifier le plus finement possible ce qui est enseigné. En effet, les RL\&E (dans une double dimension personnelle et professionnelle) impactent sur les choix didactiques des enseignants. Ce faisant, ce qui s'enseigne relève en partie de rapports à la langue, aux textes, aux dispositifs, aux activités, aux valeurs, aux visées scolaires du texte, etc. Le rapport à... est dans mon approche à la fois un outil heuristique pour analyser l'activité enseignante et un contenu de la classe de littérature. C'est la construction de cette double approche que je souhaite ici présenter en exposant à la fois le maillage théorique qui me permet d'expliciter un usage du concept et la façon dont je peux reconstruire des RL\&E. La réflexion s'organise en trois parties. La première situe les emprunts théoriques effectués aux approches cliniques et microsociologiques dans la perspective didactique qui est la mienne. La seconde traite de la conceptualisation et de la formalisation des RL\&E au sein de la didactique du français et de la littérature en abordant les questions méthodologiques qui permettent de les reconstruire. Enfin, la dernière partie traite des liens entre les RL\&E et les contenus enseignés dans la classe de littérature.

\section{La question des emprunts}

3 Je reconstruis la notion à l'intersection des travaux menés d'une part en clinique d'orientation psychanalytique, notamment en me référant aux travaux de J. Beillerot (1996) et de C. Blanchard-Laville (2013), et d'autre part en sociologie, en particulier dans le champ de la microsociologie, en prenant appui sur les travaux de B. Charlot (1997). Ces travaux ont contribué à formaliser le concept de rapport au savoir dans le domaine des sciences de l'éducation. D'un point de vue didactique, ils sont intéressants pour réfléchir à diverses dimensions du sujet didactique qu'est l'enseignant et d'éclairer sa pratique.

\section{1. Emprunts à la clinique d'orientation psychanalytique}

Pour J. Beillerot (1996), le rapport au savoir est un processus par lequel un sujet, à partir de savoirs acquis, produit de nouveaux savoirs singuliers lui permettant de penser, de transformer et de sentir le monde naturel et social. Il se réfère ainsi à une conception freudienne du rapport au désir de savoir. C. Blanchard-Laville dans la continuité de ces travaux redéfinit « le rapport au savoir comme un processus créateur de savoir pour un sujet-auteur, nécessaire pour agir et pour penser » (2013, p. 133) et analyse comment le rapport au savoir de l'enseignant s'actualise «dans l'espace de la classe en situation d'enseignement » $(2013,135)$. Son approche basée sur des récits de vie autobiographiques ou des observations filmées en classe de mathématiques l'a amenée à considérer :

qu'à l'intérieur de la situation d'enseignement, l'enseignant de mathématiques est amené à mettre en scène son propre rapport au savoir mathématique, au travers $d u$ 
discours qu'il déroule lorsqu'il s'agit d'un cours traditionnel ou au travers des activités qu'il propose et des paroles qu'il ne peut manquer de prononcer pour initier ces activités, pour en effectuer " la dévolution » aux élèves, pour catalyser leur travail, ou encore animer le groupe et éventuellement pour institutionnaliser les éléments de savoir qui émergeront de la séquence (2013, p. 135).

La réflexion qui est la mienne vise également la compréhension de tout ce que l'enseignant mobilise consciemment ou de façon non conscientisée, explicitement et implicitement dans l'actualisation de ses pratiques en classe, et ce qu'il cherche à transmettre à travers les activités qu'il propose aux élèves. En somme, il s'agit d'identifier les finalités qu'il confère à son enseignement et comment il les met en œuvre par ses intentions et son action. Pour le dire autrement encore, je cherche à identifier ce qu'il pense transmettre aux élèves à travers ses choix didactiques et pédagogiques et ce qu'il transmet de façon effective. Toutefois, l'approche didactique qui est la mienne se distingue de celle de C. Blanchard-Laville. Je ne mobilise pas la perspective clinique qui cherche à éclairer les traumatismes :

Tous ces éléments me font avancer l'hypothèse que le transfert didactique de l'enseignant, qui ne peut manquer d'être issu de son rapport traumatique au savoir (et lequel d'entre nous n'a pas un rapport traumatique au savoir?) constitue le canal par lequel l'enseignant va transmettre à son insu les caractéristiques traumatiques, les impensés, les enkystements, les fossilisations de son propre rapport au savoir (2013, p. 137).

Il m'importe de comprendre quels sont les contenus enseignés du fait des rapports des enseignants à la littérature d'une part et à son enseignement d'autre part. Cela signifie que selon moi l'activité d'enseignement est en partie conçue dans l'articulation de ces deux rapports, qui reposent sur l'expérience des lecteurs qu'ils sont devenus (de par leur gout, leur formation, etc.) et sur la façon dont ils reconstruisent les finalités de cet enseignement (entre les demandes institutionnelles et leur redéfinition du métier d'enseignant et des contextes dans lesquels ils enseignent). Les élèves de leur côté sont soumis à ces rapports et ils créeront leur propre rapport à la littérature et à son apprentissage en partie à partir de cet enseignement tel qu'ils l'auront vécu et reconstruit. Les rapports à la littérature des enseignants et des élèves peuvent faciliter des enseignements ou des apprentissages, ou au contraire révéler les difficultés que je peux reconstruire à partir des observations ou des entretiens semi-dirigés que je réalise. Il s'agit en partie de reconstruire le désir de transmettre : qu'est-ce que les enseignants partagent de la littérature? Qu'est-ce qui donne du sens à leur métier ou à leur action d'enseignement de la littérature?

\section{2. Emprunts à la microsociologie}

7 B. Charlot, quant à lui, conçoit :

le rapport au savoir (comme) le rapport au monde, à l'autre et à soi-même d'un sujet confronté à la nécessité d'apprendre ; le rapport au savoir est l'ensemble des relations qu'un sujet entretient avec tout ce qui relève de "l'apprendre » et du savoir: objet, "contenu de pensée», activité, relation interpersonnelle, lieu, personne, situation, occasion, obligation, etc., liés en quelque façon à l'apprendre et au savoir. (Charlot, 1997, p. 3)

8 Les travaux de B. Charlot et du groupe ESCOL portent davantage sur le sujet élève, mais ils éclairent le rapport que chaque sujet entretient avec soi et les autres, en particulier dans une situation didactique. Ils permettent d'entrevoir, autrement que l'approche clinique à 
orientation psychanalytique, le caractère dynamique des rapports pour chaque sujet et l'importance des interactions avec les autres. Ainsi, une situation d'enseignement et d'apprentissage peut être considérée comme une situation où les rapports de l'ensemble des sujets à l'objet enseigné se confrontent entre eux. Ils peuvent alors évoluer dans cette confrontation ou au contraire résister à toute transformation, en fonction des sujets. Ainsi, si de nombreux travaux en sociologie ont éclairé les processus de démobilisation, de fuite des enseignants (Van Zanten, 2001 ; Van Zanten, Grospiron, Kherroubi et Robert, 2002) ou encore de deuil de la discipline (Barrère, 2002), dans la recherche portant sur les enseignants impliqués en milieu populaire dirigée par B. Monfroy $(2007)^{1}$, il apparait à travers les entretiens que le rapport à la littérature permet en partie à ces enseignants de ne faire le deuil ni de l'élève ni de l'étudiant de lettres qu'ils ont été. Ils trouvent au contraire dans leurs expériences de lecteurs et probablement d'élèves les leviers pour enseigner une discipline dont ils repensent les finalités et les moyens. Ils peuvent ainsi aisément faire le deuil d'une discipline rêvée et adapter leur enseignement autrement qu'en terme de renoncement (Monfroy, Jellab, Carion, Dias-Chiaruttini, Mortier, 2007).

9 L'approche microsociologique de B. Charlot permet, par ailleurs, de dépasser le déterminisme de l'approche sociologique de P. Bourdieu (1965) pour qui le rapport au savoir construit dans les familles détermine le rapport aux savoirs des élèves. Sans nier l'impact de ce facteur, l'approche de B. Charlot permet de considérer que chaque sujet élabore son rapport au savoir à travers un maillage plus complexe d'expériences vécues, d'aspiration, d'intention, de volonté, de désir et de rejet. L'éducation familiale y joue un rôle, mais elle n'est pas exclusive.

10 Si l'approche microsociologique est une référence importante dans ma reconstruction de la notion, je m'en distingue sur au moins deux points. Le sujet que j'analyse est un enseignant (et non un élève). De plus, je ne m'intéresse pas au savoir en général, mais aux objets de savoir liés à un enseignement spécifique qui relèvent de ce qui, à travers les degrés d'enseignement, est désigné par littérature. Cela signifie que je considère que dans une perspective didactique les rapports aux savoirs scolaires varient selon les disciplines, voire même selon les composantes de la discipline. C'est ainsi que lors mes premiers travaux (Dias-Chiaruttini, 2007) j'ai recouru à une formulation différente, évoquant des rapports aux savoirs disciplinaires en les différenciant des rapports aux savoirs professionnels pour en éclairer la complémentarité (Monfroy et al., 2007). Depuis, je cherche à comprendre le rapport à la littérature que les enseignants ont construit ainsi que le rapport à son enseignement qu'ils construisent. Je m'intéresse aux savoirs, aux vécus, aux expériences, aux intentions des enseignants, etc. qui leur permettent d'enseigner ce qu'ils désignent par littérature. Quelles sont les connaissances et les expériences à partir desquelles les enseignants fondent leur conception de la littérature et de son enseignement? Que mobilisent-ils de ces connaissances et expériences dans leur activité d'enseignement?

11 Ces deux références, clinique et microsociologique, sont importantes dans l'usage que je propose du concept, notamment en ce qui concerne ma reconstruction du sujet didactique. La première permet en effet de penser le sujet didactique enseignant comme un sujet psychique dont une partie de l'action est implicite, non consciente, mais déterminante de son action d'enseignement singulière. La seconde replace ce sujet dans sa dimension individuelle et interrelationnelle et permet de concevoir le rapport à la littérature et à son enseignement comme un processus dynamique. 
12 Cette reconstruction du sujet didactique montre à quel point l'activité enseignante est complexe à saisir. Je ne reconstruis du sujet et de sa pratique que ce que mes choix méthodologiques permettent de reconstruire à partir de ce à quoi chaque enseignant me permet d'accéder.

\section{Finalités et choix méthodologiques des RL\&E}

\section{1. Une approche singulière des RL\&E} dimension : la dimension personnelle des pratiques de lecture de la littérature (visant le sujet social qu'est l'enseignant, l'élève et l'étudiant qu'il a été) et la dimension professionnelle qui prend en compte sa formation professionnelle, le contexte d'enseignement dans lequel il exerce, ses pratiques déclarées, mais aussi ses pratiques effectives observées, la façon dont il redéfinit son métier et les finalités de la littérature (le sujet enseignant). Je considère que ces deux dimensions (sujet social, sujet enseignant) sont constituantes du sujet didactique qu'est un enseignant. Toutefois, je cherche moins à construire les relations entre ses dimensions que l'impact des rapports à la littérature et à son enseignement (RL\&E) sur ce qui est enseigné. Autrement dit, dans mon approche, l'analyse des RL\&E n'a d'intérêt que celui d'éclairer une pratique d'enseignement de la littérature. C'est d'ailleurs ce qui m'amène à recourir au pluriel. Chaque sujet construit des rapports à la littérature selon les sphères sociales, selon les activités, selon les situations dans lesquelles il est impliqué.

En cela, mes travaux sont assez proches de ceux de J. Emery-Bruneau (2010). Je partage avec l'auteure québécoise l'idée que « les pratiques d'enseignement liées à cet objet sont aussi hétéroclites que les conceptions rangées sous l'étiquette "littérature"sont polysémiques » (Bruneau-Emery, 2014, p. 72). Comme elle, je considère que les rapports à la littérature relèvent d'une synergie ou d'une dynamique pour reprendre son expression. Toutefois, contrairement à son approche, je ne pose pas de conception théorique a priori de ce qu'est la littérature. C'est à partir de la reconstruction des rapports des enseignants à cet objet et à son enseignement que je peux en partie caractériser ce que les enseignants désignent, déclarent enseigner et enseignent - en analysant leurs pratiques - sous le nom de littérature. Cependant, ce qui est enseigné dans mon approche est reconstruit par un croisement de deux types de données qui dépendent à la fois des observations de situations et des déclarations des enseignants sur leurs conceptions, leurs visées et leurs pratiques déclarées de cet enseignement.

\section{2. Des choix méthodologiques}

Cette approche induit des choix méthodologiques. Je construis mes données à travers des questionnaires ou des entretiens semi-directifs pour reconstruire des déclarations de pratiques et des observations filmées (dont la caméra est toujours orientée vers l'enseignant) pour appréhender des pratiques effectives.

Toutefois, il ne s'agit pas pour moi de confronter des déclarations de pratiques et des observations de pratiques. Si d'un point de vue clinique les tensions entre le déclaratif et l'activité effective permettent d'analyser des zones de conflits, de souffrance ou de traumatisme, d'un point de vue didactique, il s'agit uniquement de deux façons très 
contrastées d'accéder aux pratiques enseignantes. Aucune ne me semble d'ailleurs plus fiable qu'une autre, chacune répond différemment à des questions de recherche. Cela signifie que je considère que les pratiques observées ne permettent pas plus que les pratiques déclarées de construire une approche plus fiable de la réalité de la classe de littérature. Je ne hiérarchise pas ces méthodologies selon un axe de fiabilité ou de pertinence. Elles permettent de reconstruire une réalité contrastée de la classe de littérature. Selon la question de recherche posée, l'une ou l'autre de ces méthodologies peut être plus pertinente, mais aucune ne serait en soi plus pertinente qu'une autre. Dans les deux cas, c'est toujours une reconstruction de pratiques de classe. Dans le premier, il s'agit du discours d'un sujet enseignant qui évoque et décrit ses pratiques telles qu'il les pense dans une situation de communication qui peut prendre la forme d'un entretien ou d'un questionnaire. Dans le second, il est question d'une reconstruction par l'observation d'un sujet chercheur plus ou moins impliqué dans la situation qu'il observe pour répondre à sa question de recherche. Ce ne sont ainsi pas les différences ou les ressemblances entre ces reconstructions de pratiques qu'il m'intéresse d'analyser, mais comment ensemble elles permettent d'éclairer ce qui s'enseigne. Cela signifie que je considère que les données construites à l'aide de l'un et de l'autre de ces deux choix méthodologiques peuvent s'éclairer et aider à l'interprétation des données pour construire des résultats. Les différences que je peux observer entre le dire et le faire sont constitutives de la complexité des pratiques dont je cherche à retracer une intelligibilité qui permet d'éclairer ce qui s'enseigne.

17 Par ailleurs, la prise en compte du contexte social où les enseignants exercent est importante : elle permet d'éclairer la façon dont les rapports à la littérature évoluent du fait des conceptions de son enseignement. Le choix des textes incarne le processus même de transmission de textes et des valeurs que les enseignants leur confèrent. Ainsi, cette enseignante de cycle 3 qui a grandi dans un milieu modeste - dit-elle - et qui enseigne dans une école appliquée ${ }^{2}$ déclare mobiliser sa bibliothèque intérieure et évoque lors de son entretien des lectures incontournables :

Oui ce sont des incontournables ++ attends-moi je me souviens du jour où on m'a lu La chèvre de monsieur Seguin ++ bon ce sont des lectures qui t'ouvrent le monde de la lecture + la petite fille aux allumettes / bon j'en ai pleuré / après ça tu lis autrement et puis je te l'ai dit je veux faire bouger des représentations de lecture gnangnan des contes ${ }^{3}$

18 Il va de soi que ces représentations de la lecture sont les siennes et celles qu'elle suppose que ses élèves pourraient avoir. Un autre enseignant, exerçant également au cycle 3, dans une école très hétérogène sur le plan social, explique pour quelles raisons, selon lui, les corpus patrimoniaux sont à réviser : «Non, mais le patrimoine est souvent d'un lassant pas possible! Je vous dis là on gagnerait à utiliser des textes plus récents et plus objectifs $»^{4}$. Pour une autre enseignante de collège situé en milieu très défavorisé, les textes incarnent la résilience. Elle conçoit la littérature comme « un refuge pour échapper à ce qui nous fait mal ++ ce qui manque à mes élèves c'est cette capacité à fuir le réel, à rêver pour mieux affronter la vie ».

19 Pour cette autre enseignante exerçant également dans un collège situé dans une ville industriellement dévastée par le chômage, les textes littéraires visent une réconciliation avec la littérature et au-delà avec l'école et la vie. Elle déclare choisir des textes de B. Lavilliers autour du travail à la chaine à l'usine. Elle pense ainsi permettre à ses élèves de retrouver, redécouvrir l'histoire de leur famille et pouvoir alors éprouver la beauté du texte, de la langue et s'en saisir pour parler d'eux-mêmes : 
L'élève est un tout ++ et euh en même temps c'est un truc rare et je crois que le métier d'enseignant ne peut se concevoir que par rapport à ça ++ parce qu'en même temps / ça influe sur tes choix de textes // ça influe sur ta dimension euh ::: quand je vois le choix des textes que j'utilise / que je vais travailler /// on va travailler sur l'usine / on va travailler sur des gens qui bossent à la chaine (...) et en même temps les textes que j'ai choisis pour le regroupement de textes // y a un texte de Lavilliers xxx parce que je me dis que ça ressemble aussi sinon à nos élèves /, mais peut être à leurs parents / à leurs grands-parents / que je crois aussi qu'il faut parler des choses qu'ils connaissent parce qu'en même temps ça leur permet de mieux rentrer dans ce que l'on essaie de leur apprendre ++ et seulement après on pourra leur amener des choses beaucoup plus complexes ou des choses qui ne connaissent pas et que c'est une démarche intellectuelle que l'on apprend c'est-àdire partir de ce que je sais faire et si je sais bien le faire et je vais oser aller plus loin et finalement c'est voilà + osons + osons un petit peu toujours plus et puis aussi // oser aussi en classe euh / ben se permettre un petit peu de sortir des sentiers battus et tout en respectant parce que moi je pense qu'on peut tout à fait respecter les textes // les programmes / s'attacher à respecter les programmes c'est important ${ }^{5}$.

Ces discours éclairent la singularité des pratiques qui sont toujours contextualisées et imbriquées dans des conceptions multiples concernant les contenus disciplinaires, mais aussi les élèves, leur capacité d'apprentissage, et la façon dont les enseignants redéfinissent leur métier: le sens qu'ils donnent à leur action. B. Lavilliers n'est pas seulement lu dans cette classe parce que les élèves sont issus d'un milieu ouvrier. Il est aussi lu parce que l'enseignante a pour projet de partager la lecture qu'elle fait de ce texte et les valeurs qu'il véhicule avec ses élèves. Elle pense que la langue de Lavilliers les aidera à exprimer leur propre rapport au monde. Ce texte viendra ainsi côtoyer des extraits choisis de Germinal d'Émile Zola.

\section{3. Des tentatives de catégorisation}

Reste en suspens la question de la catégorisation des RL\&E. Faut-il ne produire que des études de cas ou tenter de construire des typologies? La construction de ces dernières est toujours méthodologiquement délicate et en soi aucune construction n'est satisfaisante. Elles réduisent la richesse des singularités au risque parfois de survaloriser certains aspects plutôt que d'autres. Toutefois, le travail d'analyse permet de dégager un type de RL\&E plus dominant que d'autres chez un enseignant en fonction des questions du chercheur. Il faut bien considérer que ce tri de rapports différents est toujours la reconstruction du chercheur dans le cadre d'un questionnement singulier.

Ainsi, je dirais que toute typologie relève d'une tentative de clarification des résultats construits et vise la clarification de systèmes de cohérence et d'interprétation des données. C'est par conséquent moins les limites des processus de typologie dont il faut se garder que les critères qui fondent les types de rapports reconstruits.

Par ailleurs, certains travaux ont mis en avant des catégories de profils, de postures d'enseignement de la littérature (De Beaudrap, 2004 ; Langlade, 2016, entre autres) souvent sans mobiliser la notion de rapport à... ou sans construire la notion. À ma connaissance aucun travail de synthèse ne confronte ces approches qui peuvent même s'ignorer entre elles. Pourtant, elles portent bien souvent sur les pratiques d'enseignants stagiaires du secondaire, encore en formation, et éclairent autant des entrées contrastées dans le métier que des modalités d'enseignement très différentes. Toutefois, les 
catégories retenues pour décrire les pratiques enseignantes renvoient bien souvent à des reconstructions de catégories de lecteurs issus des travaux sur la réception: des approches subjectives vs des approches d'experts (par exemple: De Beaudrap, 2004). Jamais ces travaux n'explicitent la construction théorique de ces catégories. Ainsi, G. Langlade (2016) évoque 3 catégories de postures dominantes dans le choix des œuvres des enseignants stagiaires: didactique ; spécialiste et celle de l'amateur. Il regrette qu'une quatrième posture, pédagogique, "très fréquente chez les enseignants expérimentés ne se retrouve pas ici, sans doute du fait du manque de pratique et de recul des stagiaires. » (p. 142-143). D'où viennent ces catégories? Qu'est-ce qui justifie le recours à des catégories qui semblent préexister aux données? En quoi est-ce éclairant d'une expérience professionnelle qui pèse sur les pratiques et les postures, et sans doute sur des RL\&E ? Les articles font peu état de ce souci méthodologique, pourtant il est garant de la pertinence des catégories construites, mobilisées, réempruntées. Qu'éclairent les théories littéraires des pratiques effectives? Quels en sont les apports et les limites? Par ailleurs, ces travaux imbriquent les notions entre elles sans véritablement expliciter les liens qui semblent ainsi se reconstruire.

Ce constat éclaire la nécessité d'un travail de clarification des notions et de leurs usages, pour observer la mise en garde que formulaient I. Delcambre et Y. Reuter (2002) au sujet de la diversité des termes pour rendre compte des rapports à l'écriture des élèves : «En l'absence d'une limite ou d'une construction précise des composantes et de leur articulation, on court le risque d'une dilution conceptuelle » (p. 15).

\section{RL : un contenu enseigné}

La notion de RL\&E, en se focalisant sur le pôle enseignant, se distingue des concepts tels que les représentations des élèves, leur clarté cognitive ou encore des usages qui peuvent être faits du rapport à..., notamment du rapport à l'écriture. Ces concepts sont liés aux apprentissages des élèves et peuvent expliquer leurs difficultés d'apprentissage. Cependant, la configuration conceptuelle de la notion de conscience disciplinaire partage des liens importants avec l'usage que je propose de RL\&E. Cette notion permet d'analyser " la manière dont les acteurs sociaux et plus particulièrement les acteurs scolaires (re)construisent les disciplines scolaires» (Reuter, 2007, p. 57). Y. Reuter (2003) l'avait dans un premier temps conçue pour analyser la reconstruction des disciplines scolaires par les élèves. Néanmoins la notion est heuristique pour analyser les rapports des enseignants aux disciplines scolaires et l'impact sur leurs pratiques. Sur ce point les travaux de J. Van Beveren (2014) (voir aussi Dumortier et Van Beveren, 2010) sont assez éclairants. Toutefois si les deux notions (RL\&E et conscience disciplinaire) gagneraient à être articulées, elles se différencient, du moins dans l'usage que j'en fais, sur un point essentiel. Je ne considère pas la littérature comme une discipline scolaire, y compris au lycée. Ainsi, analyser la conscience disciplinaire du français d'un enseignant revient en partie à prendre en considération ses rapports et conceptions de la littérature et de son enseignement, mais ni la conscience disciplinaire ni la discipline scolaire ne se réduisent à cet enseignement. C'est une approche tronquée de la conscience disciplinaire des enseignants que j'analyse puisque j'opère un découpage des contenus enseignés. Toutefois je place bien cet enseignement au sein d'une discipline sur laquelle j'interroge les enseignants, comme on peut le voir dans l'extrait suivant d'un entretien mené avec une enseignante de cycle 3 : 
- et ma conception de la discipline ? Non faire du français ou de la littérature ce n'est pas la même chose d'ailleurs le français c'est plus vraiment la discipline enseignée

- on n'enseigne plus le français?

- on enseigne la langue et la culture / le patrimoine de la langue française, mais la discipline français comme j'enseignais avant avec mes questionnaires / mon heure de +++ et de ++ ça c'est fini ++, mais je fais autant qu'avant, mais autrement et surtout j'accompagne mes élèves avant / c'était aux élèves de me suivre et tu vois certains n'allaient pas loin / et je ne pouvais pas faire autrement avec mon questionnaire $^{6}$

Par ailleurs, la conscience disciplinaire résulte en partie des enseignements reçus, mais elle n'est pas un contenu de la classe. Or, je considère que les rapports à la littérature sont un contenu qui se transmet à travers l'action d'enseignement. Ainsi, lorsqu'une enseignante de français exerçant dans un lycée se réclame de « l'agrégation de l'être ${ }^{7}$ » et qu'elle déclare ne pas chercher à faire lire les classiques à ses élèves, mais à leur permettre « d'éprouver » la tragédie à travers le jeu des textes, son RL\&E s'articule à la conscience disciplinaire qu'elle a de la discipline qu'elle enseigne qui donne du sens à son action et à ses choix d'enseignement. Ce qu'elle enseigne est aussi un rapport aux textes littéraires qui se différencie du rapport distancié au texte que vise l'enseignement de la littérature au lycée.

Les RL\&E influencent les contenus enseignés et en formalisent certains. Ils permettent par conséquent de mieux comprendre les contenus qui émergent dans la classe de littérature. Dans l'extrait ci-dessous, l'enseignant lors d'un débat interprétatif amène les élèves à élaborer un questionnement au-delà de la fabula du texte. Ces situations assez récurrentes dans mon corpus de débats interprétatifs au cycle 3 de l'école élémentaire m'avaient amenée à considérer que l'erreur interprétative ou la surinterprétation du texte caractérisent certaines pratiques enseignantes en fonction du rapport à la littérature et de la façon dont les enseignants conçoivent cet enseignement. Pour cet enseignant, qui a une formation initiale scientifique, le texte littéraire est le lieu d'un raisonnement, il ne s'agit pas tant de comprendre le texte que d'apprendre à raisonner et à questionner à partir d'un texte. C'est ce rapport au raisonnement qu'il enseigne et qui dans certains cas peut conduire à des malentendus sur le texte :

Marine : on a aussi vu de qui est-ce qu'il était inspiré le livre

Enseignant : oui et c'était?

Marine : Memphis Slim

Enseignant: Memphis Slim oui et on s'est posé une question d'ailleurs / qu'est-ce que c'était ? quelle question on s'est posée à ce sujet-là ? Estelle

Estelle : qui est le fils Slim

Enseignant : qui était le fils Slim et puis euh::: et il me semble que vous vous étiez posé une question à laquelle on n'avait pas su répondre

Stéphane : est-ce que c'est Little Lou?

Enseignant : est-ce que Memphis Slim c'était Little Lou ? et est-ce que Little Lou et il y avait plusieurs hypothèses? est-ce que c'est Memphis Slim?

Claire : est-ce que Little Lou / c'est l'auteur?

Enseignant : est-ce que Little Lou c'est l'auteur ou est-ce que // c'est une dernière hypothèse?

Maeva : ou est-ce que c'est un personnage inventé ?

Enseignant : ou est-ce que c'est un personnage inventé ? bon là on n'a pas vraiment d'éléments pour répondre à ça ++ après ++ allons avancez ${ }^{8}$

28 En fait le texte est juste « inspiré » de la vie de quelqu'un, mais l'enseignant ne traite pas du sens de cet énoncé et invite les élèves à poser des questions par rapport au fait que 
c'est un livre inspiré de la vie de quelqu'un. Ainsi, ce qui est ici enseigné n'est pas une compréhension de ce texte, mais une façon de se questionner à partir des textes littéraires qui prend source dans le RL\&E de cet enseignant. Ce faisant ce raisonnement sur le texte est bien un contenu enseigné ainsi que le rapport à la littérature qui le permet.

Les RL\&E contribuent au travail d'interprétation des pratiques observées sans poser $a$ priori une norme ou une référence qui permettrait d'objectiver la pertinence de ces RL\&E. Je ne cherche pas à savoir si certains rapports sont plus "efficaces» ou plus "pertinents» que d'autres, ce serait alors modifier la visée compréhensive de ma démarche pour envisager une démarche de recherche qui se centrerait sur l'évaluation des pratiques enseignantes. Je ne suis pas sure que dans cette perspective les RL\&E soient une donnée intéressante. Par contre, ils trouvent une place des plus pertinentes en formation pour que chaque sujet éclaire ses pratiques et explicite des choix qui parfois pourraient échapper au sujet enseignant lui-même. Dans l'extrait précédent, l'utilisation du texte qui est faite ne relève pas d'une incompréhension du texte de la part de l'enseignant (ce serait une interprétation abusive de la part du chercheur), mais d'un usage qui prend sens parmi les conceptions qu'il a de la littérature et les objectifs qu'il se fixe. C'est un élément à prendre en considération pour la formation des enseignants, ce ne sont pas tant des gestes (comme les gestes d'étayage par exemple) qu'il convient de travailler que les RL\&E qui induisent telle utilisation du texte et pèsent sur l'activité enseignante.

\section{En guise de conclusion}

Beaucoup de questions restent en suspens à l'issue de cette présentation de mon usage des rapports RL\&E et à la façon dont je reconstruis et analyse les pratiques enseignantes. Toutefois cette réflexion éclaire le nécessaire travail de maillage des emprunts et de clarification de ce qui est spécifique dans un usage singulier. Cette spécificité découle en partie à la fois du champ de recherche dans lequel chaque chercheur se situe et de son questionnement. Ainsi les RL\&E me semblent particulièrement heuristiques pour comprendre - en partie - ce qui s'enseigne sous le nom de littérature, à travers les degrés d'enseignement, et comment ils permettent de penser la formation des enseignants au plus près des pratiques en construction. D'autres usages pourront éclairer les limites de mon approche et éclairer des apports qui m'échappent...

\section{BIBLIOGRAPHIE}

Barré-de Miniac, C. (2000). Le rapport à l'écriture. Villeneuve-d'Ascq : Presses universitaires du Septentrion.

Barré-de Miniac, C. (2002). Le rapport à l'écriture. Une notion à plusieurs dimensions. Pratiques, 113-114, 29-39. 
Barrère, A. (2002). Les enseignants au travail, routines incertaines. Paris : L'Harmattan.

Beillerot, J. (dir.), (1996). Pour une clinique du rapport au savoir. Paris : L'harmattan.

Blanchard-Laville, C. (2013). Du rapport au savoir des enseignants, Journal de la psychanalyse de l'enfant (Vol. 3), 123-154.

Charlot, B. (1997). Du rapport au savoir, Éléments pour une théorie. Paris : Ed. Anthropos.

De Beaudrap A.-R. (dir.) (2004). Images de la littérature et de son enseignement. Étude réalisée à l'IUFM des Pays de la Loire auprès des PLC de Lettres. Nantes : CNDP-CRDPP.

Dias-Chiaruttini, A. (2007). Le rapport aux savoirs disciplinaires des enseignants en milieu populaire : un outil pour comprendre le travail didactique de l'enseignant?, Colloque international AIRDF, Didactique $\mathrm{du}$ français : le socioculturel en question, Université de Lille 3 - Villeneuve-d'Ascq, du 13 au 15 septembre 2007. En ligne : http://airdf.ouvaton.org/archives/lille-2007/PDF/Dias\%20Chiaruttini \%20A\%2017.pdf

Dias-Chiaruttini, A. (2015). Le débat interprétatif dans l'enseignement du français. Berne : Peter Lang.

Dias-Chiaruttini, A. (2016). Æuuvres patrimoniales à l'école primaire : la place singulière des fables. In Denizot N., Dufays J.-L. et Ulma D. (dir.). Les fables à l'école : perspectives didactiques. (pp. 141-152). Namur : Presses universitaires de Namur.

Dumortier, L.-L. et Van Beveren, J. (2010). Les sources du savoir des futurs professeurs de français du secondaire supérieur en Belgique francophone, Repères 42, 45-61.

Émery-Bruneau, J. (2010). Le rapport à la lecture littéraire. Des pratiques et des conceptions de sujetslecteurs en formation à l'enseignement du français à des intentions didactiques. Thèse de doctorat, Québec : Université Laval.

Falardeau, É. et Simard, D. (2007). Rapport à la culture et approche culturelle de l'enseignement. Canadian Journal of Education / Revue canadienne de l'éducation, Vol. 30, No. 1, 1-24.

Falardeau, É. et Simard, D. (2011). La culture dans la classe de français : Témoignages d'enseignants. Québec : Les Presses de l'Université Laval.

Monfroy, B., Jellab, A., Dias-Chiaruttini, A, A., Carion, P. et Mortier, L. (2007) Travail enseignant et construction de postures professionnelles en milieu populaire : rapport aux savoirs, savoirs mobilisés et engagement, Rapport de recherche $\mathrm{N}^{\circ} \mathrm{R} / \mathrm{RIU} / 06 / 07$ : En ligne : http://www.lille.iufm.fr/IMG/pdf/ Travail_enseignant_et_construction_de_postures_professionnelles_en_milieu_populaire_ rapport_aux_savoirs_savoirs_mobilises_et_engagement.pdf

Reuter, Y. (2003). La représentation de la discipline ou la conscience disciplinaire. La Lettre de la DFLM, $\mathrm{n}^{\circ}$ 32, Namur, diffusion AIRDF, 18-22.

Reuter, Y. (2007). La conscience disciplinaire, Éducation et didactique, vol 1 - $\mathrm{n}^{\circ}$ 2, 55-71.

Reuter, Y. (dir.) (2007/2010/2013). Dictionnaire des concepts fondamentaux en didactiques. Bruxelles, De Boeck.

Van Beveren, J. (2014). La discipline scolaire " français » : du questionnement identitaire à la conscience disciplinaire. Thèse de doctorat soutenue à l'université de Liège, Belgique, sous la direction de J.L. Dumortier.

Van Zanten, A. (2001). L'école de la périphérie. Scolarité et ségrégation en banlieue. Paris : PUF.

Van Zanten, A., Grospiron, M.-F., Kherroubi, M. et Robert, A. (2002). Quand l'école se mobilise. Paris : Éditions La Dispute. 


\section{NOTES}

1. Il s'agissait d'une recherche pluridisciplinaire regroupant des sociologues (Brigitte Monfroy et Aziz Jellab) et des didacticiens de disciplines différentes (mathématique, histoire-géographie et français), intitulée : Travail enseignant et construction de postures professionnelles en milieu populaire : rapport aux savoirs, savoirs mobilisés et engagement.

2. Les écoles appliquées en France sont rattachées aux ESPE (École Supérieure du professorat et de l'éducation), les enseignants qui y sont nommés sont tous formateurs et bénéficient d'une décharge d'enseignement pour la formation.

3. Propos recueillis lors des entretiens semi-directifs menés sur les pratiques du débat interprétatif (Dias-Chiaruttini, 2015). Les propos sont retranscrits en respectant en partie les conventions proposées par Vion (1992), en particulier pour signaler les pauses.

4. Propose recueillis par questionnaires dans le cadre d'une recherche exploratoire sur les pratiques déclarées des textes du patrimoine (Dias-Chiaruttini, 2016). Vion (1992), en particulier pour signaler les pauses.

5. Propos recueillis lors d'un entretien semi-directif mené dans la recherche sur les rapports aux savoirs disciplinaires des enseignants impliqués en secteur défavorisé (Dias-Chiaruttini, 2007).

6. Propos recueillis lors des entretiens semi-directifs menés sur les pratiques du débat interprétatif (Dias-Chiaruttini, 2015).

7. Propos recueillis dans le cadre de la recherche sur le rapport aux savoirs disciplinaires (DiasChiaruttini, 2007).

8. Situation de débat interprétatif filmé dans le cadre de la recherche sur l'émergence de ce genre disciplinaire (Dias-Chiaruttini, 2015).

\section{RÉSUMÉS}

Cet article revient sur une approche singulière du concept «rapport à ». Formalisé en psychologie et en sociologie, il a permis en didactique du français le développement de travaux importants, notamment sur les pratiques d'écriture des élèves ou encore sur les pratiques d'enseignement de la littérature. C'est dans cette seconde perspective que le concept est ici mobilisé pour rendre compte de ces apports, des questions méthodologiques qu'il soulève, et de la façon dont il peut être articulé à d'autres notions pour éclairer ce qui s'enseigne en classe de littérature.

This article presents a singular approach to the concept of «relation to». Formalized in psychology and sociology, it allowed in French didactics the development of important works, particularly on the writing practices

of students or on the teaching practices of literature. It is in this second perspective that the concept is mobilized here to relate these contributions, the methodological questions it raises, and how it can be articulated with other notions to brighten what is taught in class of literature. 
INDEX

Mots-clés : rapport à, enseignement de la littérature, pratiques enseignantes, didactique du français

Keywords : relation to, teaching of literature, teaching practices, didactics of French

\section{AUTEUR}

\section{ANA DIAS-CHIARUTTINI}

Maitre de conférences, Université de Lille SHS, CIREL 4354 